\title{
A Predictive Model for Antioxidative Regulation of Wheat under Cadmium Stress
}

\author{
Siquan $\mathrm{Hu}^{1, \mathrm{a}^{*} \text {, Sisi Zhao }}{ }^{1, \mathrm{~b}}$ and Haiou Wang ${ }^{2, \mathrm{c}}$ \\ ${ }^{1}$ School of Computer and Communication Engineering, University of Science and Technology \\ Beijing, Beijing, 100083, China \\ ${ }^{2}$ School of Chemical and Biological Engineering, University of Science and Technology Beijing, \\ Beijing, 100083, China \\ ahusiquan@ustb.edu.cn, bguaiguaisi@163.com, wanghaiou@ustb.edu.cn
}

\begin{abstract}
Keywords: Cadmium, Prediction, ROS, Signal transduction, Oxidative stress
Abstract. Cd-induced signal transduction triggers ROS production, which promotes oxidative stress indirectly. In the present work, we aggregated and interpreted the abundant biological evidence that has been accumulated to date into a network, and then constructed a multi-level dynamic model of signal transduction during Cd-induced ROS changing based on the network. We applied this model to predict ROS changes of plant under different Cd stress levels, and it turned out to be that our model well predicted the shifting trend of ROS of plant exposed to different $\mathrm{Cd}$ levels. The model could be useful on directing future experiments.
\end{abstract}

\section{Introduction}

Cd-induced elevated generation of reactive oxygen species (ROS) in cells plays a pivotal role in the development of $\mathrm{Cd}$ toxicity symptoms [1]. ROS is an inevitable product of plants' metabolism. In normal cases, it will be constantly cleared with the synergistic effect of antioxidative systems, so that the ROS production and defense can keep balance. However, marked ROS accumulation will be dangerous which can produce severe damages affecting cell viability, and even lead to cell death [2]. Till date, there are lots of studies related to the effect of heavy metal $\mathrm{Cd}$ on plants' growth and physiological. Yet there are limitations existing in the current studies and experimentation. Most of previous works just focus on one or a few specific kinds of plants' responses related to specific kinds of elements contributing to Cd tolerance [3]. However, much less has been done on a systems level to synthesize all existing evidence into a network model of Cd-induced ROS changing.

In this work, we combine the existing theory with the abundance of experimental results regarding ROS generation and its regulation, constructing a dynamic modelling of the Cd stimulated ROS changing process and the signal transduction cascades between different kinds of plants. This model recapitulates a comprehensive array of known behaviors and phenotypes. Since the model is made up of node-level information, this agreement serves as validation [4]. The model enables an unprecedented understanding of the Cd-induced ROS changing and reveals aspects of the system, particularly in the conversion among different states of $\mathrm{Cd}$, where critical experimental evidence is lacking.

\section{Model Construction}

The Construction Of The Cd-induced ROS Changing Signal Transduction Network. The first step in building the dynamic model is to construct the regulatory network that represents the system. A network is an abstraction of a system in which each element is represented as a node, and the relationship between each pair of interaction or regulation is represented by an edge. Edges in signal transduction networks are generally directed and signed. The components involved in ROS changing include proteins, the metabolic processes, some important inorganic compounds, certain biological processes and entities. Positive edges in our network correspond to activation, up-regulation, or biochemical synthesis, and are represented with a terminating arrowhead, while negative edges indicate deactivation, inhibition, or consumption, and are shown as terminating in a solid circle. 


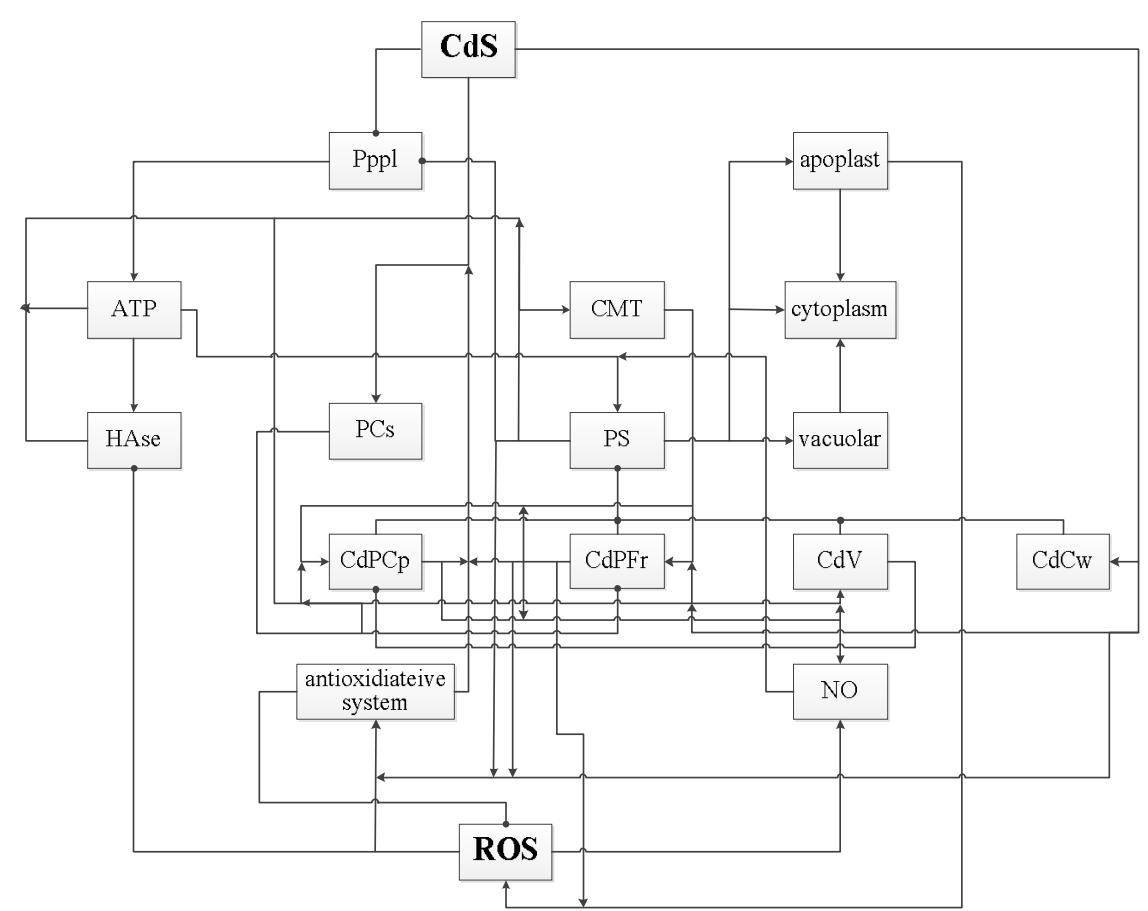

Fig. 1 Current knowledge of Cd-induced ROS changing model.

The resulting network is constituted by 17 nodes and 49 edges in Fig. 1. A brief description of the biology represented by the network is as follows: Like the other heavy metals, Cd can affect the biochemical and physiological activities of plants through a variety of ways. It interferes with normal metabolism of cells, leads to a chain of disorders and lacking of water of the plant. Meanwhile, Cd stress will cause diminished photosynthetic activity [5], thus preventing the production of ATP and $\mathrm{H}^{+}$-ATPase. And then, protein synthesis will be inhibited; the number of metal transporter protein and stability of membrane will be influenced [6]. The application of $\mathrm{Cd}$ attributes to generation of ROS and plants try to force this by stimulation of antioxidants defense system [7].

In the model, $\mathrm{CdS}$ represents the Cd concentration in solution; because of the restriction of cell wall, there will be part of $\mathrm{Cd}$ bound to the cell wall (i.e. $\mathrm{CdCw}$ ); due to the cellular detoxification mechanisms, part of the $\mathrm{Cd}$ that enters the cell will be combined with PCs to form complex (i.e. CdPCp); some heavy metal complexes will be transported into vacuole (i.e. CdV) for cellular compartmentalization, and the rest of the $\mathrm{Cd}$ that have entered the cell will exist in a free state (i.e. $\mathrm{CdPFr}$, which will cause a significant increase in membrane lipid peroxidation products and do harm to the cells.

\section{Model Evaluation}

The Model Predicts The Effects Of Different Levels Of Cd Stress On ROS. We investigated the effect that different levels of Cd have on ROS changing. ROS is normally produced in the cellular systems at low level by plant in order to perform its physiological function. Fig. 2 (A) shows the situation of ROS changes without external $\mathrm{Cd}$. From it, we can see that in our model the ROS level fluctuates around 2 which indicates a balance between ROS production and defense in normal cases. The equilibrium level 2 is determined by the regulation of each component of the multiple dynamic model, thus can be used as ROS initial state of our model with Cd stress. From Fig. 2 (B), we can see that when $\mathrm{Cd}$ concentration was low $(\mathrm{CdS}=4)$, ROS rises to about 2.42 for a brief period, then it was quickly regulated to normal state and fluctuated around 2, after that ROS fell slowly until it eventually maintained at about 1.62. With the rising $\mathrm{Cd}$ concentration $(\mathrm{CdS}=6,10)$, from Fig. 2 (C), we can obviously find that the value of ROS presented a trend of rising with fluctuations. Besides, with a higher concentration of $\mathrm{Cd}$, the simulation curve fluctuates less and the ROS level increased more quickly which indicated a higher oxidation degree of the plants. When concentration of Cd exceeds the maximum that the plant can tolerate, it appeared the situation shown in Fig. 2 (D): the value of ROS 
rose rapidly to its peak, and then declined sharply to 0 . In addition, a higher concentration of $\mathrm{Cd}$ could lead to a more quickly approach to the peak level, and a faster declining to 0 . We suspect that the plants will soon be oxidized to death at such a high concentrations of $\mathrm{Cd}$.

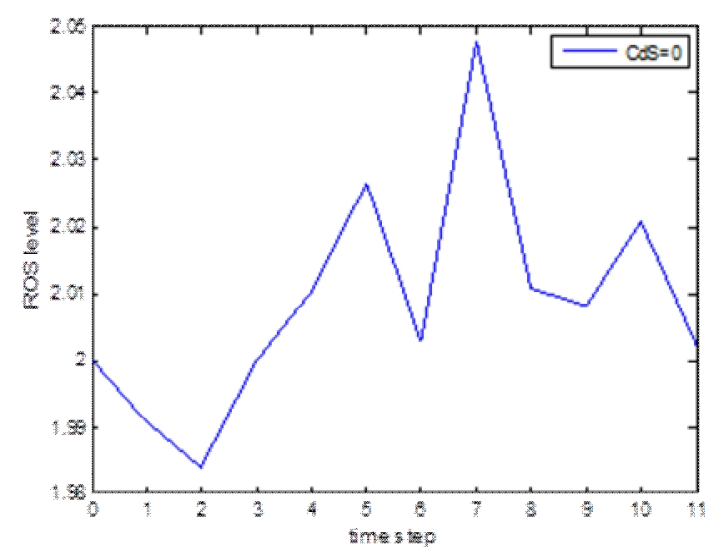

A
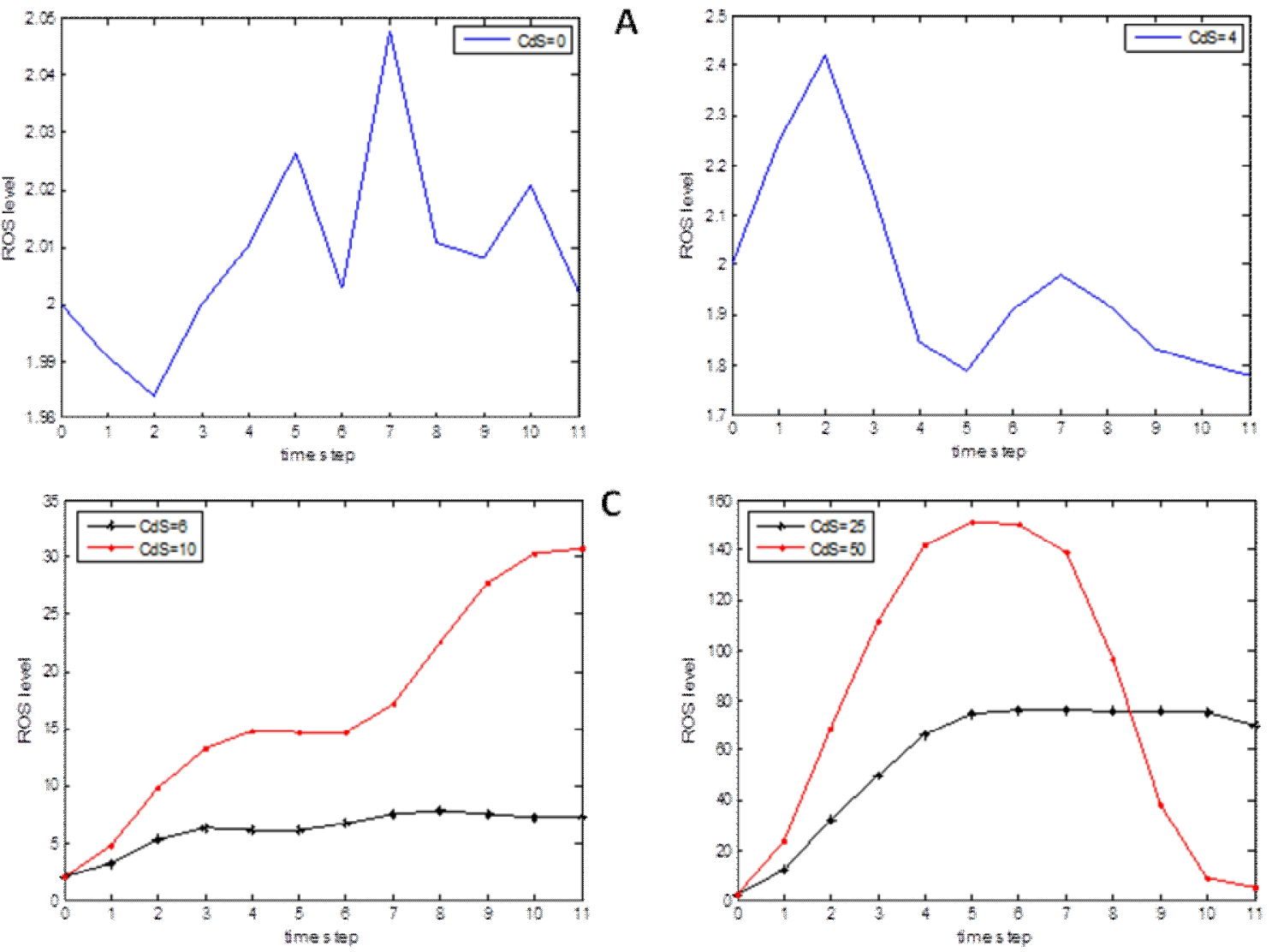

C

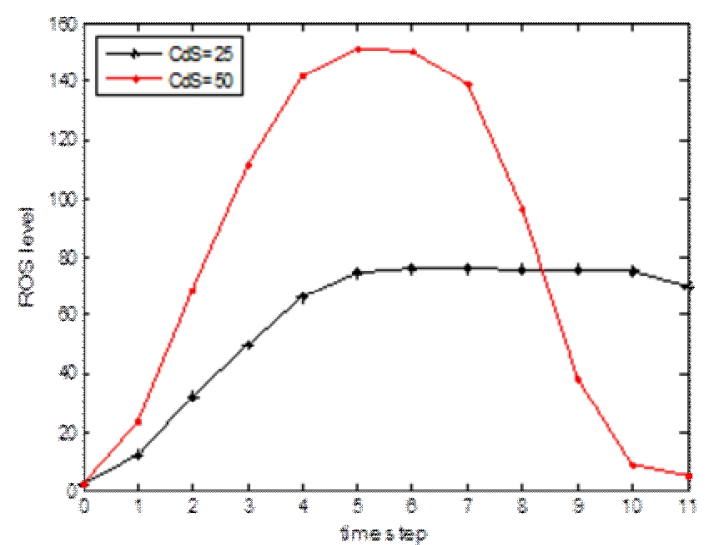

B

D

Fig. 2 ROS changes at different Cd concentration.

Experimental Test Of The Effect Of Cd On ROS. In order to test the validity of the model, we experimentally assessed the effect of $\mathrm{Cd}$ stress in wheat roots and shoots. The wheat was grown from seeds and watered with deionized water every day. Approximately 360 healthy and young fully-expanded leaflets from a month old wheats were selected for Malondialdehyde measurements. They were divided into 3 groups and treated with modified Hoagland's nutrient solutions which contained $0,10 \mathrm{mg} / \mathrm{L}$ and $100 \mathrm{mg} / \mathrm{L} \mathrm{Cd}$ solution respectively. The MDA content was estimated according to the method of Hodges [8].
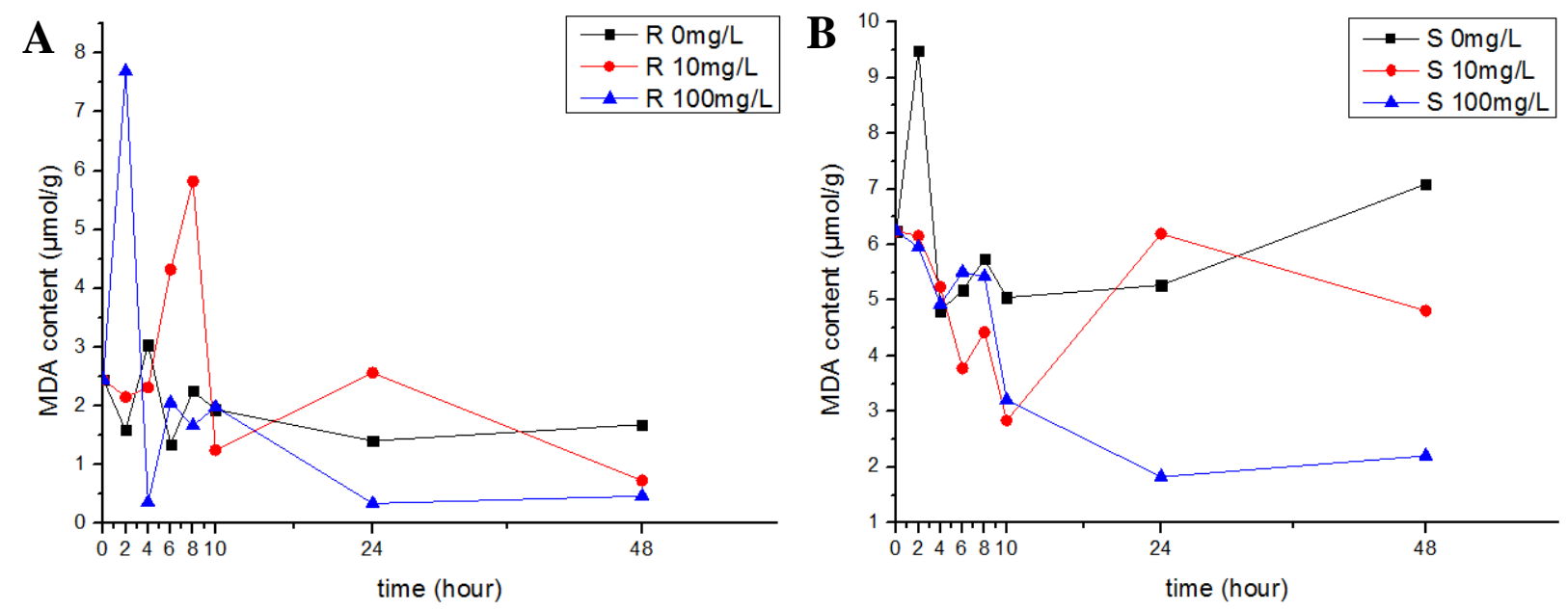

Fig. 3 The experimental results of MDA changing of wheat exposed to different concentrations of Cd $(0,10 \mathrm{mg} / \mathrm{L}, 100 \mathrm{mg} / \mathrm{L})$. (A) MDA changing of wheat roots. (B) MDA changing of wheat shoots. 
The results of the experiments were shown in Fig. 3. The MDA value of wheat roots and shoots both tended to be stable in the control group. The MDA value of roots fluctuated around 2 and that of shoots fluctuated around 5.5. Under the Cd stress of $10 \mathrm{mg} / \mathrm{L}$, the MDA value of wheat roots firstly had a short rising to about 5.8, which was 2 times more than that of the controls, and then it fell slowly with fluctuations until it eventually maintained at about 1 , while the MDA value of wheat shoots firstly increased with fluctuations to about 6.2, and then decreased sharply. Both of the MDA value of wheat roots and shoots when they reached a relatively stable state were lower than that of the controls. When the $\mathrm{Cd}$ concentration approached $100 \mathrm{mg} / \mathrm{L}$, the MDA value of wheat roots and shoots both tended to decline with fluctuations, yet the MDA value of wheat roots had a 200\% rapidly increase compared to that of the controls within the first two hours.

Comparing the model results with experimental result under different concentrations of $\mathrm{Cd}$ in solution, we can see that the ROS trends at different degrees of Cd stress predicted by our model are consistent with the experimental results: (1) At low concentration of Cd, the value of ROS may have a transitory increase in the beginning, but after a period of time, it will fall down and even be lower than the equilibrium value of normal state due to a chain of regulations. This kind of low concentration of $\mathrm{Cd}$ even promotes the growth for plants. (2) Under the treatment of high $\mathrm{Cd}$ concentration, we find that ROS levels rose with fluctuations as the experiment time increasing, and it even gets much higher than that of the controls.

\section{Summary}

A multi-level dynamic model is constructed to synthesize the extensive existing evidence into a network of Cd-induced ROS changes. The model can simulate ROS changes of plant under different $\mathrm{Cd}$ stress levels, and then we compared the predictions with the experimental results. The model well predicted the shifting trend of ROS of plant exposed to different $\mathrm{Cd}$ levels. In addition, our model made up for the limitation of the experiment operations from the setting of $\mathrm{Cd}$ concentrations and sampling frequency, which enhanced the prediction range and improved the accuracy on a systems level.

\section{Acknowledgements}

The study was supported by the National Natural Science Foundation of China (No. 21007003).

\section{References}

[1] Benavides M P, Gallego S M, Tomaro M L. Cadmium toxicity in plants[J]. Brazilian Journal of Plant Physiology, 2005, 17(1): 21-34.

[2] Sharma I. Arsenic induced oxidative stress in plants[J]. Biologia, 2012, 67(3): 447-453.

[3] Arasimowicz-Jelonek M, Floryszak-Wieczorek J, Gwóźdź E A. The message of nitric oxide in cadmium challenged plants[J]. Plant science, 2011, 181(5): 612-620.

[4] Sun Z, Jin X, Albert R, et al. Multi-level modeling of light-induced stomatal opening offers new insights into its regulation by drought[J]. 2014.

[5] Ekmekçi Y, Tanyolac D, Ayhan B. Effects of cadmium on antioxidant enzyme and photosynthetic activities in leaves of two maize cultivars[J]. Journal of plant physiology, 2008, 165(6): 600-611.

[6] Singh P, Shah K. Evidences for reduced metal-uptake and membrane injury upon application of nitric oxide donor in cadmium stressed rice seedlings[J]. Plant Physiology and Biochemistry, 2014, 83: 180-184.

[7] Rady M M, Hemida K A. Modulation of cadmium toxicity and enhancing cadmium-tolerance in wheat seedlings by exogenous application of polyamines[J]. Ecotoxicology and environmental safety, 2015, 119: 178-185. 
[8] Hodges D M, DeLong J M, Forney C F, et al. Improving the thiobarbituric acid-reactive-substances assay for estimating lipid peroxidation in plant tissues containing anthocyanin and other interfering compounds[J]. Planta, 1999, 207(4): 604-611. 